\title{
Comparative Study of Computer Vision Syndrome and Smart Devices among the Employers and Students in College of College of health and Medical Technique of University of Al-Furat Al-Awsat Techniques. Kufa
}

\author{
Shukria shadhan chyad ${ }^{a}$ Atheer Hussein zyara ${ }^{b}$ Saad Saleem Raheem ${ }^{c}$ and Ahmed Takleef Al- \\ Hasani $^{\mathrm{d}}$ \\ ${ }^{a}$ College of Health and Medical Technology /kufa, Al-Najaf, Iraq. \\ ,${ }^{b}$ Department of Community Health /College of Health and Medical Technology /kufa Al-Najaf, Iraq. \\ ${ }^{c}$ Department of Community Health/College of Health and Medical Technology/kufa Al-Najaf, Iraq. \\ ${ }^{d}$ College of Health and Medical Technology /kufa Al-Najaf, Iraq.
}

ª Drshalq@yahoo.com bwwatheer@yahoo.comc pharmasaad@yahoo.com ${ }^{d}$ Ahmedpct17@gmail.com.

Submission date:- 7/5/2017 Acceptance date:- 7/12/2017 Publication date:- 6/8/2018

Keywords: Computers, Smart devices, Employers, Students, Risk factors.

\begin{abstract}
Objectives Survey about numbers of students and employers that they were influenced by recurrent using of computers and smart devices, in addition to that, the comparative study performing by knowing of relation ship with different and effect on them. Materials And Methods This study was executed on individuals of students and emploees during 1 year only (from January $1^{\text {st }} 2016$ to August $15^{\text {th }} 2017$ ) in the college of Health and Medical Technology /kufa in province of Al-Najaf . It is included the relation between frequent using of computers and smart devices and other risk factor. Results The results shown significant differences in student's users of computers and smart devices in which there were little numbers of visions influencing among them in different ages and genders. Utilizing for long periods during days in different purposes yet didnt have greater effect on users in case of computer vision syndrome according to the employer's users. Since the preparation of students were having medical glass in male and female and the letter has rates more than the male. In other hand the ages at about (24-32) the using of computers and smart devices was, and the more risk factors among all of them had have greater rates of correlation with the glass wearing in a group of students that they have eye allergy in comparison with the time user. Also these results were shown the superiority of male in comparison with female in using these devices So, from the results mentioned above the convergence in numbers of students that they have vision affects due to employing of these devices in such of ages about [(19-23), (2432)] in male and female, but the first ages have slightly higher rates than other)] by percentage (50\%) of the students that suffer from computer vision syndrome from all proportion, whereas $2^{\text {nd }}$ group of another ages has low significant differences in comparison with previous by the percentage closely (36.36\%). The employer's users of computer and smart devices have significant differences $(\mathbf{p} \leq \mathbf{0 . 0 5})$ from the previous results, where it was noticed large numbers of users suffer from problems in their vision due to profuse utilization for long periods of these advices. At the ages (3140) using for different purposes whether (science, entertaining or both of them) has close correlation with hitting of employers by vision syndrome, where this factor has significant differences in comparison with other risks $(\mathbf{p} \leq \mathbf{0 . 0 5})$, while utilizing of these devices under different lights numbers appeared affect lower than it, also these results were shown no significant differences with regard to employer's users that they were utilized optical glass after they had using these devices, also effect of this factor ( light numbers) on the putting of eyeglass and presence of significant
\end{abstract}


differences $(\mathbf{p} \leq \mathbf{0 . 0 5})$ in comparison with the individuals of users that laying screen protection . We found significant differences $(\mathbf{p} \leq \mathbf{0 . 0 5})$ in numbers of employers were wearing eyeglass after utilizing these appliances and these demonstrate the vulnerability of devices users and the percentage was (58.14\%). Conclusions : Using of computers and smart devices of students more than employers. Appearing different enfluencing of eye glass wearing of students and employers that utilizing different computers and smart devices with some of risk factors according to others. It is Found assuring correlations among different risk factors, but shown in employers greater than students.

\section{1- Introduction}

The generic name of "Computer vision syndrome", it is defined by the American Optometric Association as a complex of eye and vision problems related to the activities which stress the near vision and which are experienced in relation, or during, and the symptoms of computer vision syndrome may vary depending on several factors which includes amount of time spend, viewing distance, seating posture, level of computer screen, and underlying visual acuity disturbances if any [1]. Treatment of computer vision syndrome involves proper identification of the etiologic factors and correction of visual errors if existent. Epidemiologic studies on physical exposure during computer use have mainly focused on the average duration of exposure [2]. Special attention should be paid to ergonomic factors like correct posture in the chair, lighting arrangement, antiglare screen on the computer and establishing proper working habits. During the last two decades, the number of workers with visual display units (VDU) has increased dramatically. In 2001, approximately 65\% of the Swedish workforce used a VDU in their occupation, compared to $30 \%$ in 1989 [3]. Since the late 1980s, the use of non-keyboard input devices have increased rapidly and today the market is filled with a large number of different non-keyboard input devices, although the most widely used is still the computer mouse [4]. Early 20 th century has seen increasing use of computers worldwide for both professional and personal use. This has also resulted in a drastic change in the educational sector, resulting in advert use of this technology for instruction in schools and universities. In the present era of excessive and rampant computer usage, there has been an upsurge of computer related health problems. Ocular complaints of computer users have been grouped together and collectively termed as computer vision syndrome (CVS) [5]. A complex of various environmental work factors characterizes computer work, and there are several featuresthat are relevant when discussing the development of musculoskeletal problems in this type of work. Somephysical and psychosocial factors may be specific for computer work, while others can also be present in occupational groups with no computer use. An example of generic factors concerning computer work can be illustrated byprolonged sitting, postures in the neck and hand intensive work [6]. The focus on cumulative exposure originates from the Cinderella theory. In this theory, lowforce demands during computer use lead to continuous activity of small muscle fibers, presumed to be active all the time. This continuous activity is believed to cause tissue damage over time [7]. Repetitive work has been associated with an increased risk of musculoskeletal symptoms of the wrist and forearm [8], [9],[10]. With exposure to both extreme postures and repetitive tasks it has been suggested that the risk increases, compared with exposure to only one risk factor [8]. Extensive computer use is often associated with symptoms of the upper extremity; several reviews have found a positive relation between the duration of computer use and the occurrence of upper-extremity symptoms [11],[12]. The adverse health effects on eyes include asthenopic symptoms such as eyestrain, tired eyes, irritation, redness, blurred vision and double vision [13], [14]. The forces applied to the computer mouse and keyboard may be a risk factor for musculoskeletal symptoms [15]. It has been observed that $3-4 \mathrm{~h}$ of computer mouse work could lead to fatigue in the muscles of the forearm [16]. It is not known if the forces applied to the sides and button of the computer mouse is associated with increased risk for developing musculoskeletal symptom. It has been observed that subjects with more severe musculoskeletal symptoms apply higher force while keyboarding [10]. Several hypotheses have been proposed for the pathogenesis of work-related musculoskeletal symptoms and pain [17],[18. One suggests that low static contraction during work may result in a recruiting pattern or motor programme, in which only type I muscle fibers are used, and this may lead to selective motor unit fatigue and damage [15]. Asimilar hypothesis known as the 'Cinderella hypothesis' has been proposed by Ha "gg [19]. Eye symptoms and visual discomfort have been associated with VDU work [20].The viewing of digital electronic screens is no longer restricted to desktop computers located in the workplace. Today's visual requirements may include viewing laptop and tablet computers, electronic book readers, smartphones and other electronic devices either in the workplace, at home or in the case of portable equipment, in any location. Furthermore, computer use is not restricted to adults [21]. Positive results from improved visual conditions and optometric corrections have been demonstrated in a 6-year follow-up study [22]. Current guidelines regarding monitor placement at VDUs suggest that the top of the screen should be at or slightly below eye level. In recent years, lower monitor placements have been proposed [23]; however, there is not enough scientific evidence available to change the current guidelines. The lack of recovery time after muscle activity also seems a risk factor for the occurrence of muscle or tissue damage [24]. Then, it is not so much a high mean exposure of computer use causing damage, but a high number of days with prolonged duration of exposure, implying an insufficient recovery time within a day. Another possibility is that, for example, typing very fast or clicking the mouse very frequently could cause musculoskeletal damage, especially if the recovery time is insufficient 
[25]. Then, the high number of days with this style of high frequency input device use could be an important risk factor.

Objectives Survey about numbers of students and employers that they were influenced by recurrent using of computers and smart devices, in addition to that correlation of them with the different risk factors.

\section{2- Materials And Methods}

This study was designed as cross section and executed on individuals were divided into two groups, the first group included [(117 students; male 48 , female 69$)$ ], consisted of the $3^{\text {rd }}$ stages of this college, the $2^{\text {nd }}$ group included [(60 individuals; 40 male, 20 female)], all of them were using computers during 1 year (from January $1^{\text {st }} 2016$ to August $15^{\text {th }}$ 2017). This study included the relation between frequent using of computers and smart devices and other risk factor. These data were analyzed statically with SPSS 8.0 statistical package (ANOVA- analysis of variance - two ways analyze; Mean $\pm \mathrm{SE})$. (P) values less than or equal to 0.05 has been evaluated as statistically significant[26].

\section{3- Results And Discussion}

The results were shown significant differences in students users of computers and smart devices in which there were little numbers of visions influencing among them in different ages and genders and these results were found scales with the expeling of [1]. In spite of employing for long periods during days in different purposes [(59+41.76)] didnt has greater effect on the eye infection and they were agreement with previous study of [2], for example at age about (19-23) appearing significant differences in screen distance from eye $[(\mathbf{5 9} \pm \mathbf{1 4 . 4 8})]$ whereas social states has $[(\mathbf{5 9} \pm \mathbf{4 6 . 7 3})]$, but these were disagreement with previous study of [25] that demonstrate Physical factors, psychosocial and organisational factors as well as individual factors are all thought toaffect the workers musculoskeletal health, also they have been investigated in relation to computer work and upper extremity and neck symptoms, and it is believed that factors like time pressure and high perceived work load interact in the development of the symptoms, but [23]corrorates our study resulkts regarding effect of screen distance on the eye influencing. These results also had appeared superiority of female in the using in comparison with male $[(\mathbf{4 5} \pm \mathbf{3 1 . 8 4}, \mathbf{1 4} \pm \mathbf{9 . 8 9})]$ respectively, and all of risk factors have the similarity affects in both of them and this evidence hadn't any significant differences and this quid little significant correlation among different risk factors and eye affects, since the preparation of students were having eye glass in male and female $[(\mathbf{4 . 0 0} \pm \mathbf{2 . 8 3}),(\mathbf{1 0 . 0 0} \pm \mathbf{7 . 0 7}) ; \mathbf{1 4} \pm \mathbf{9 . 0 9})]$, respectively and the letter has rates more than the male. In other hand the ages at about (24-32) the using of computers and smart devices was [(58+68.63)], and the more risk factors among all of them had greater rates of correlation with the glass wearing in a group of students that they have eye allergy $[(\mathbf{5 8} \pm \mathbf{2 9 . 0 7})]$ in comparison with the time user $[(\mathbf{5 8} \pm \mathbf{6 8 . 6 3})]$. Also these results were shown the superiority of male in comparison with female in using these devices $[(\mathbf{3 4} \pm \mathbf{2 4 . 0 5}),(\mathbf{2 4} \pm \mathbf{4 4 . 5 8})$, so we found the differences in effectiveness in the gender due to varying in pharmacokinetic, pharmacodynamic and pathologicalm reseans [27]. The results mentioned above the convergence in numbers of students that they had vision affects due to employing of these devices in such of ages about [(19-23), (24-32)] in male and female, but the first ages have slightly higher rates than other $[(\mathbf{1 4 . 0 0 \pm 9 . 9 0 )}, \mathbf{( 7 . 0 0 \pm 4 . 9 4 )}]$ by percentage $(\mathbf{5 0 \%})$ of the students were suffering from computer vision syndrome from all proportion, whereas $2^{\text {nd }}$ group of another ages has low significant differences in comparison with previous $[(\mathbf{1 1 . 0 0 \pm 7 . 7 7 )}, \mathbf{( 4 . 0 0 \pm 2 . 8 2 )}]$ by the percentage closely $(\mathbf{3 6 . 3 6 \%})$, in which these results agreement with previous study of [28],[29] proved the several risk factors were including gender have strong correlation and influencing on vision problems syndrom. [Table 1]. 
Table (1). Numbers of students of the $3^{\text {rd }}$ stages of studying had used computers and smart devices.

\begin{tabular}{|c|c|c|c|c|c|c|c|c|c|c|}
\hline \multicolumn{2}{|c|}{ Age(yrs.) } & \multicolumn{8}{|c|}{ Students } & \multirow{3}{*}{ Total } \\
\hline Influnced & & & $19-23$ & & & & 24-32 & & & \\
\hline \multicolumn{2}{|l|}{ Factors } & $\mathbf{m}$ & $\mathbf{F}$ & \multicolumn{2}{|c|}{ Total } & $\mathbf{M}$ & $\mathbf{F}$ & \multicolumn{2}{|c|}{ Total } & \\
\hline \multicolumn{2}{|c|}{ Gender } & $(14 \pm 9.89$ & $(45 \pm 31.84)$ & \multicolumn{2}{|c|}{$(59 \pm 41.76)$} & $(34 \pm 24.05)$ & $(24 \pm 44.58)$ & \multicolumn{2}{|l|}{$(58 \pm 68.63)$} & \multirow{15}{*}{ 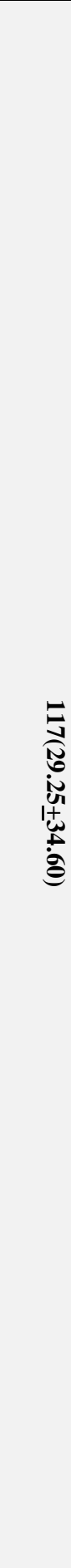 } \\
\hline \multirow{4}{*}{$\begin{array}{c}\text { Time } \\
\text { user(yrs.) }\end{array}$} & $<1$ & $\begin{array}{c}(1.00 \pm 0 . \\
70)\end{array}$ & $(4.00 \pm 2.83)$ & $\begin{array}{c}(5.00 \pm 3 . \\
53)\end{array}$ & \multirow{4}{*}{ 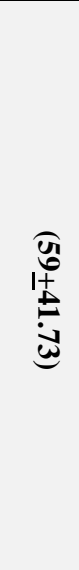 } & $(0.00 \pm 0.00)$ & $(0.00 \pm 0.00)$ & $\begin{array}{c}(0.00 \pm 0 \\
00)\end{array}$ & \multirow{4}{*}{ 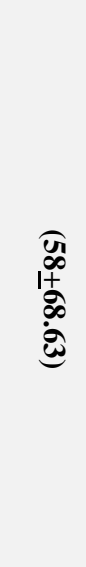 } & \\
\hline & 2 & $\begin{array}{c}(1.00 \pm 0 . \\
70)\end{array}$ & $(4.00 \pm 2.83)$ & $(5.00 \pm 3$ & & $(2.00 \pm 1.41)$ & $\begin{array}{c}(3.00 \pm 29.7 \\
3)\end{array}$ & $\begin{array}{c}(5.00 \pm 31 \\
.14)\end{array}$ & & \\
\hline & 3 & $\begin{array}{c}(0.00 \pm 0 \\
00)\end{array}$ & $(10.00 \pm 7.07)$ & $\begin{array}{c}(10.00 \pm 7 \\
.07)\end{array}$ & & $(7.00 \pm 4.95)$ & $(1.00 \pm 0.70)$ & $\begin{array}{c}(8.00 \pm 5 . \\
65)\end{array}$ & & \\
\hline & $>4$ & $\begin{array}{c}(12.00 \pm 8 \\
.49)\end{array}$ & $(27.00 \pm 19.11)$ & $\begin{array}{c}(39.00 \pm 2 \\
7.60)\end{array}$ & & $\begin{array}{c}(25.00 \pm 17 \\
69)\end{array}$ & $\begin{array}{c}(20.00 \pm 14 . \\
15)\end{array}$ & $\begin{array}{c}(45.00 \pm 3 \\
1.84)\end{array}$ & & \\
\hline \multirow{2}{*}{ Social state } & $\mathrm{S}$ & $\begin{array}{c}(13.00 \pm 9 \\
.20)\end{array}$ & $(42.00 \pm 7.07)$ & $\begin{array}{c}(55.00 \pm 1 \\
6.27)\end{array}$ & \multirow{2}{*}{ 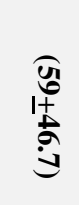 } & $\begin{array}{c}(30.00 \pm 21 \\
23)\end{array}$ & $\begin{array}{c}(22.00 \pm 15 \\
57)\end{array}$ & $\begin{array}{c}(52 \pm 36.8 \\
)\end{array}$ & \multirow{2}{*}{ 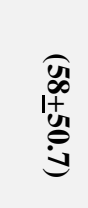 } & \\
\hline & $\mathrm{Ma}$ & $\begin{array}{c}(1.00 \pm 0 . \\
70)\end{array}$ & $(3.00 \pm 29.73)$ & $\begin{array}{c}(4.00 \pm 30 \\
.43)\end{array}$ & & $(4.00 \pm 2.83)$ & $(2.00 \pm 1.41)$ & $\begin{array}{c}(6.00 \pm 13 \\
.97)\end{array}$ & & \\
\hline \multirow{2}{*}{ Adress } & $\mathrm{U}$ & $\begin{array}{c}(13.00 \pm 9 \\
.20)\end{array}$ & $(39.00 \pm 27.60)$ & $\begin{array}{c}(52 \pm 36.8 \\
)\end{array}$ & \multirow{2}{*}{ 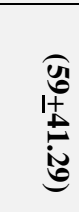 } & $\begin{array}{c}(31.00 \pm 21 \\
94)\end{array}$ & $\begin{array}{c}(21.00 \pm 14 . \\
86)\end{array}$ & $\begin{array}{c}(52 \pm 36.8 \\
)\end{array}$ & \multirow{2}{*}{ 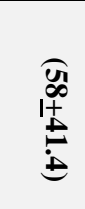 } & \\
\hline & $\mathrm{R}$ & $\begin{array}{c}(2.00 \pm 1 \\
41)\end{array}$ & $(5.00 \pm 3.53)$ & $\begin{array}{c}(7.00 \pm 4 . \\
49)\end{array}$ & & $(3.00 \pm 2.12)$ & $(3.00 \pm 2.12)$ & $\begin{array}{c}(6.00 \pm 4 . \\
24)\end{array}$ & & \\
\hline \multirow{3}{*}{ Day/week } & 1 & $\begin{array}{c}(0.00 \pm 0 . \\
00)\end{array}$ & $(15.00 \pm 10.61)$ & $\begin{array}{c}(15 \pm 10.6 \\
1)\end{array}$ & \multirow{3}{*}{ 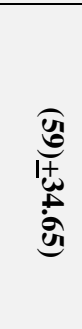 } & $(1.00 \pm 0.70)$ & $(3.00 \pm 2.12)$ & $\begin{array}{c}(4.00 \pm 2 . \\
82)\end{array}$ & \multirow{3}{*}{ 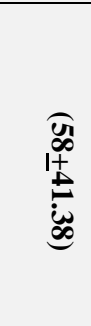 } & \\
\hline & 2 & $\begin{array}{c}(11.00 \pm 0 \\
.70)\end{array}$ & $(5.00 \pm 3.53)$ & $\begin{array}{c}(16+4.23 \\
)\end{array}$ & & $\begin{array}{c}(11.00 \pm 7.7 \\
8)\end{array}$ & $(1.00 \pm 0.70)$ & $\begin{array}{c}(12.00 \pm 8 \\
.48)\end{array}$ & & \\
\hline & $>3$ & $\begin{array}{c}(13.00 \pm 9 \\
.20)\end{array}$ & $(15.00 \pm 10.61)$ & $\begin{array}{c}(28+19.8 \\
1)\end{array}$ & & $\begin{array}{c}(22.00 \pm 15 . \\
57)\end{array}$ & $\begin{array}{c}(20.00 \pm 14 \\
15)\end{array}$ & $\begin{array}{c}(42 \pm 29.7 \\
2)\end{array}$ & & \\
\hline \multirow{2}{*}{ hrs./day } & $<12$ & $\begin{array}{c}(12.00 \pm 8 \\
.49)\end{array}$ & $(38.00 \pm 26.90)$ & $\begin{array}{c}(50 \pm 35.3 \\
9)\end{array}$ & \multirow{2}{*}{ 尊 } & $\begin{array}{c}(28.00 \pm 19 . \\
82)\end{array}$ & $\begin{array}{c}(21.00 \pm 14 . \\
86)\end{array}$ & $\begin{array}{c}(49 \pm 34.6 \\
8)\end{array}$ & \multirow{2}{*}{ 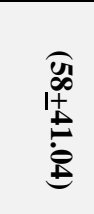 } & \\
\hline & $>13$ & $\begin{array}{c}(2.00 \pm 1 \\
41)\end{array}$ & $(7.00 \pm 4.95)$ & $\begin{array}{c}(9.00 \pm 6 . \\
36)\end{array}$ & & $(6.00 \pm 4.24)$ & $(3.00 \pm 2.12)$ & $\begin{array}{c}(9.00 \pm 6 . \\
36)\end{array}$ & & \\
\hline Purposes & scientific & $\begin{array}{c}(2.00 \pm 1 \\
41)\end{array}$ & $(4.00 \pm 2.83)$ & $\begin{array}{c}(6.00 \pm 4 . \\
24)\end{array}$ & i & $(5.00 \pm 3.53)$ & $(6.00 \pm 4.24)$ & $\begin{array}{c}(11.00 \pm 7 \\
.77)\end{array}$ & 㣽 & \\
\hline
\end{tabular}




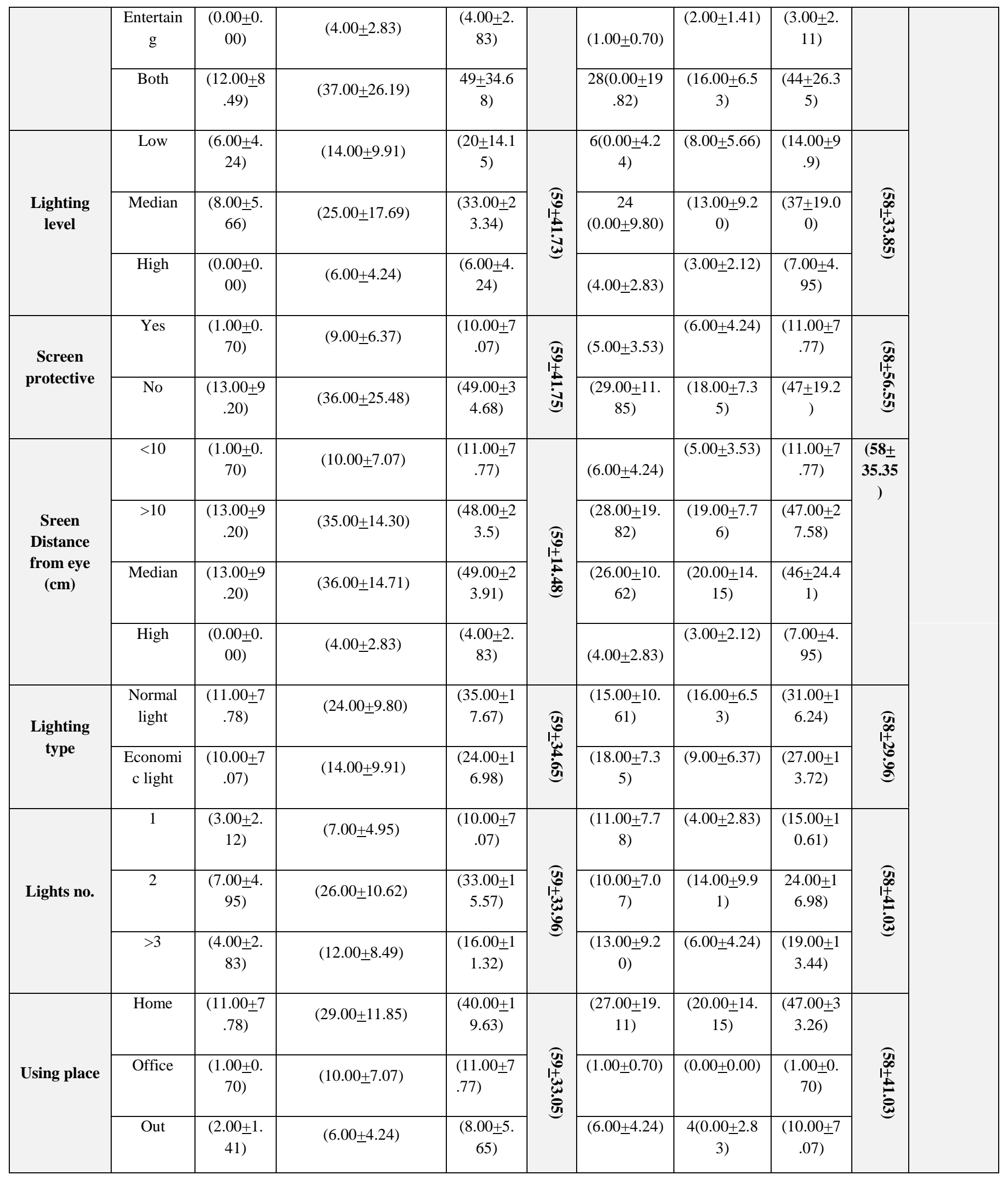




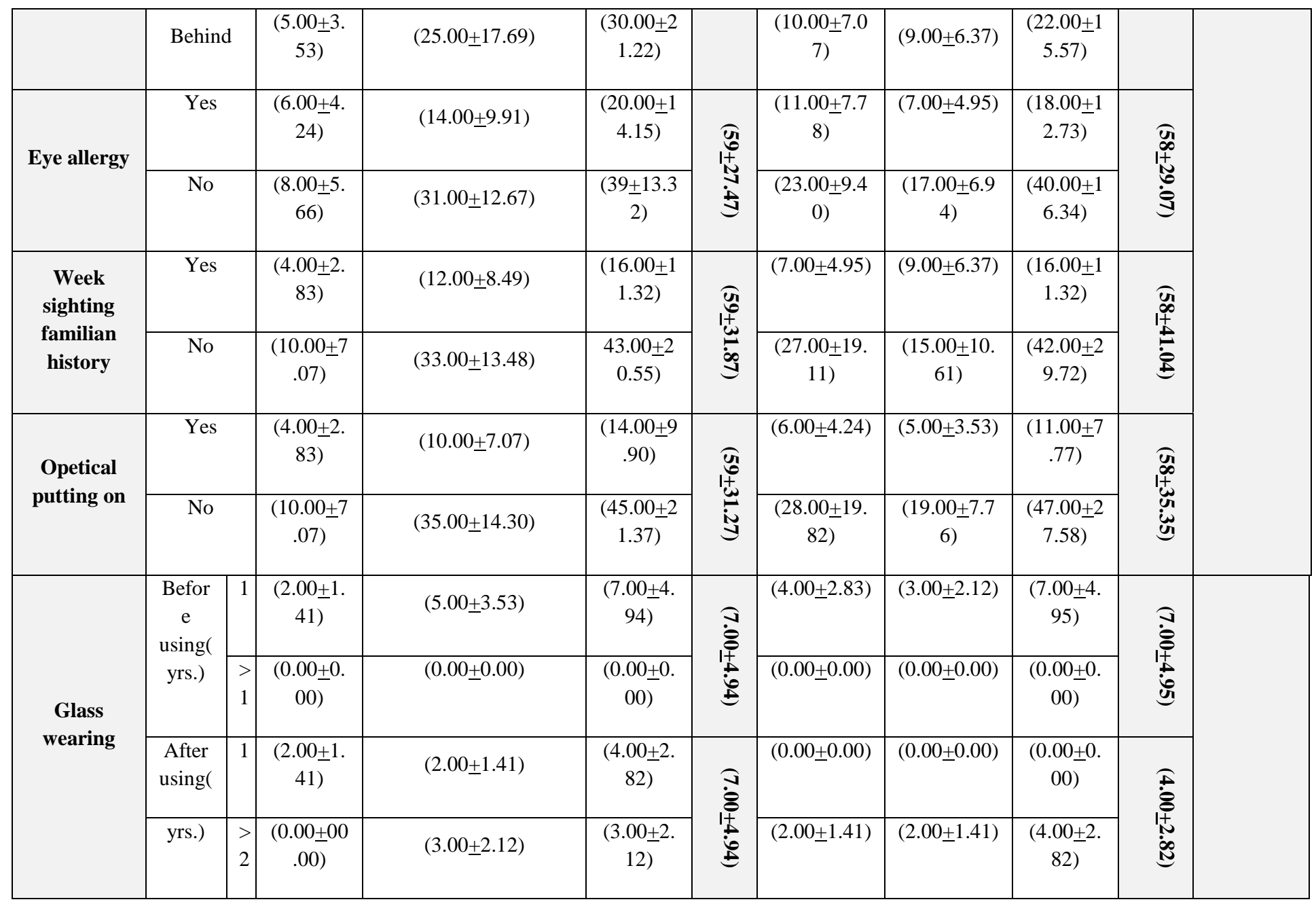

- m:male;f:female;u:urban;r:ruler;s:single;ma:marriage

- Different capital refer to significant differences between groups horizontally $(\mathrm{P} \leq 0.05)$.

\section{4-The values represent Mean $\pm \mathrm{SE}$}

Whereas the results in [Table 2] of the employer's users of computer and smart devices have significant differences $(\mathbf{p} \leq \mathbf{0 . 0 5})$ from the previous results, where it was noticed large numbers of them suffer from problems in their vision due to profuse utilization for long periods of these devices, for example during ages about (21-30) years old the male users have greater than female at the same ages range, also another ages (31-40) have closely have the same results

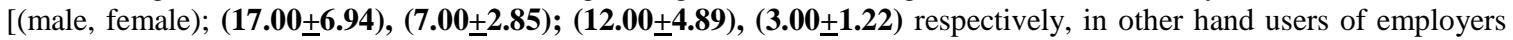

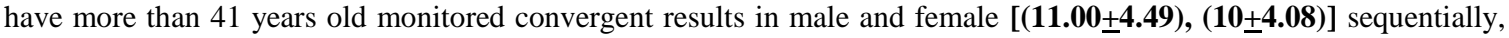
and In ages about (21-30) years were old shown using of these advices (hrs./ day) has strong relationship with the vision influencing of employers $[(\mathbf{2 4} \pm \mathbf{9 . 7 8})]$ and that mean getting significant differences between them $[(\mathbf{2 4} \pm \mathbf{6 . 6 5})$, $\mathbf{p} \leq \mathbf{0 . 0 5})]$, in comparison with the utilizing (day/ week) in which it has weaker correlation with this affect $[(\mathbf{2 4} \pm \mathbf{1 1 . 7 8})]$, these results had been proved by [7], [8] using of computers and other sifferent devices with profuse and for long periods during years for different purposes under phsychical pressures and overtimemay be lead to muscoloskeletal in eye and neck disorders. The numbers of users that they were effected $[(6.00 \pm 3.43)]$ out of $[(\mathbf{1 1 . 0 0 \pm 4 . 4 8})]$ had have glass wearing were very large proportion. Whereas at ages (31-40) using for different purposes whether (science, entertaining or both of them) $[(\mathbf{1 5} \pm \mathbf{6 . 0 8})]$ had close correlation with hitting of employers by vision syndrome $[(\mathbf{1 5} \pm \mathbf{5 . 8 8})]$, where this factor has significant differences in comparison with other risks $(\mathbf{p} \leq \mathbf{0 . 0 5})$, while utilizing of these devices under different lights numbers appeared effect lower than it, in which it was [(15 $\pm \mathbf{8 . 0 9})]$. Also these results were shown no significant differences with regard to employer's users $[(\mathbf{1 . 0 0 \pm 0 . 0 4})]$ that they were utilized optical glass after they had using these devices $[(\mathbf{5 . 0 0} \pm \mathbf{2 . 0 3}$. The employers were using computers and smart devices 
during different years $(\mathbf{> 1 , 2 , 3}$ and $>\mathbf{4})$, in which these results shown effect of this factor on the putting of eyeglass $[(\mathbf{2 1} \pm \mathbf{8 . 5 5})]$ and presence of significant differences $(\mathbf{p} \leq \mathbf{0 . 0 5})$ in comparison with the individuals of users that they were lay screen protection while working on it $[(\mathbf{2 1} \pm \mathbf{8 . 1 4})],[21]$ shown large numbers of adult users in different officers were suffering from disturbances in their working according to the newer due to getting problems in their eye, neck and hand fingers becouse profusing and strees working. We were noticed significant differences $(\mathbf{p} \leq \mathbf{0 . 0 5})$ in numbers of employers where they were wearing eye-glasses after utilizing these appliances $[(\mathbf{1 4 . 0 0 \pm 5 . 7})]$ and this demonstrates the vulnerability of device users $[(\mathbf{8} \pm \mathbf{5 . 2 4})]$ and the percentage was $(\mathbf{5 8 . 1 4 \%})$ of the proportion.

Table (2). Numbers of employer's users of computers and smart devices.

\begin{tabular}{|c|c|c|c|c|c|c|c|c|c|c|c|c|c|c|}
\hline \multirow{4}{*}{$\begin{array}{l}\text { Influnced } \\
\text { factors } \\
\text { Gender }\end{array}$} & \multicolumn{13}{|c|}{ Employers } & \\
\hline & \multicolumn{3}{|c|}{ 21-30 } & \multirow{2}{*}{\multicolumn{2}{|c|}{ Total }} & \multicolumn{2}{|c|}{$31-40$} & \multirow{2}{*}{\multicolumn{2}{|c|}{ Total }} & \multicolumn{2}{|c|}{$\begin{array}{c}\text { More than } \\
41\end{array}$} & \multirow{2}{*}{\multicolumn{3}{|c|}{ Total }} \\
\hline & & $\mathbf{M}$ & $\mathbf{F}$ & & & $\mathbf{M}$ & f & & & $\mathbf{M}$ & $\mathbf{F}$ & & & \\
\hline & & $(17.00 \pm 6.94)$ & $\begin{array}{c}(7.00 \pm \\
2.85)\end{array}$ & \multicolumn{2}{|c|}{$(24 \pm 9.79)$} & $\begin{array}{c}(12.0 \\
0+4.8 \\
9)\end{array}$ & $\begin{array}{c}(3.00 \pm 1 \\
.22)\end{array}$ & \multicolumn{2}{|c|}{$(15 \pm 6.11)$} & $\begin{array}{c}(11.00 \pm \\
4.49)\end{array}$ & $\begin{array}{c}(10.00 \pm \\
4.08)\end{array}$ & \multicolumn{2}{|c|}{$21 \pm 8.57)$} & \multirow{5}{*}{ 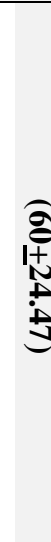 } \\
\hline \multirow{4}{*}{$\begin{array}{c}\text { Using } \\
\text { time(yrs.) }\end{array}$} & $<1$ & $(0.00 \pm 0.00)$ & $\begin{array}{c}(0.00 \pm \\
0.00)\end{array}$ & $\begin{array}{c}(0.00 \pm 0 \\
.00)\end{array}$ & \multirow{4}{*}{ 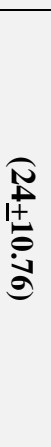 } & $\begin{array}{c}(0.00 \\
\pm 0.00 \\
)\end{array}$ & $\begin{array}{c}(0.00 \pm 0 \\
.00)\end{array}$ & $\begin{array}{c}(0.00 \pm 0 \\
00)\end{array}$ & \multirow{4}{*}{ ש্ } & $\begin{array}{l}(1.00 \pm 0 \\
.70)\end{array}$ & $\begin{array}{l}(1.00 \pm 0 \\
.70)\end{array}$ & $\begin{array}{c}(2.00 \pm 1 \\
.4)\end{array}$ & \multirow{4}{*}{$\begin{array}{l}\text { N } \\
\stackrel{N}{+1} \\
\stackrel{N}{N}\end{array}$} & \\
\hline & 2 & $(1.00 \pm 0.40)$ & $\begin{array}{l}(1.00 \pm \\
0.40)\end{array}$ & $\begin{array}{c}(2.00 \pm 0 \\
.80)\end{array}$ & & $\begin{array}{c}(1.00 \\
+0.40 \\
)\end{array}$ & $\begin{array}{c}(0.00 \pm 0 \\
.00)\end{array}$ & $\begin{array}{l}(1.00 \pm 0 . \\
40)\end{array}$ & & $\begin{array}{c}(2.00 \pm 1 \\
.81)\end{array}$ & $\begin{array}{c}(1.00 \pm 0 \\
.40)\end{array}$ & $\begin{array}{c}(3.00 \pm 2 \\
.21)\end{array}$ & & \\
\hline & 3 & $(1.00 \pm 0.40)$ & $\begin{array}{c}(2.00 \pm \\
1.81)\end{array}$ & $\begin{array}{c}(3.00 \pm 2 \\
.21)\end{array}$ & & $\begin{array}{l}(1.00 \\
\pm 0.40\end{array}$ & $\begin{array}{l}(1.00 \pm 0 \\
.70)\end{array}$ & $\begin{array}{c}(2.00 \pm 1 \\
.1)\end{array}$ & & $\begin{array}{c}(2.00 \pm 1 \\
.81)\end{array}$ & $\begin{array}{c}(0.00 \pm 0 \\
.00)\end{array}$ & $\begin{array}{c}(2.00 \pm 1 \\
.81)\end{array}$ & & \\
\hline & $>4$ & $(15.00 \pm 6.12)$ & $\begin{array}{c}(4.00 \pm \\
1.63)\end{array}$ & $\left(19 \pm \frac{7.7}{5)}\right.$ & & $\begin{array}{c}(10.0 \\
0 \pm 4.0 \\
8)\end{array}$ & $\begin{array}{c}(2.00 \pm 1 \\
.81)\end{array}$ & $\begin{array}{c}(12.00 \pm \\
5.89)\end{array}$ & & $\begin{array}{c}(6.00 \pm 2 \\
.44)\end{array}$ & $\begin{array}{c}(8.00 \pm 2 \\
.85)\end{array}$ & $\begin{array}{l}(14.00 \\
\pm 5.29)\end{array}$ & & \\
\hline \multirow{2}{*}{ Social state } & $\mathrm{S}$ & $(4.00 \pm 1.63)$ & $\begin{array}{c}(1.00 \pm \\
0.40)\end{array}$ & $\begin{array}{c}(5.00 \pm 2 \\
.03)\end{array}$ & \multirow{2}{*}{ 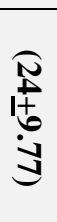 } & $\begin{array}{c}(2.00 \\
+1.81 \\
)\end{array}$ & $\begin{array}{c}(1.00 \pm 0 \\
.40)\end{array}$ & $\begin{array}{c}(3.00 \pm 2 \\
.21)\end{array}$ & (⿳) & $\begin{array}{c}(3.00 \pm 1 \\
.22)\end{array}$ & $\begin{array}{c}(1.00 \pm 0 \\
.40)\end{array}$ & $\begin{array}{c}(4.00 \pm 1 \\
.62)\end{array}$ & \multirow{2}{*}{ 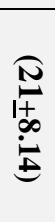 } & \\
\hline & $\mathrm{ma}$ & $(13.00+5.30)$ & $\begin{array}{c}(6.00 \pm \\
2.44)\end{array}$ & $\begin{array}{l}(19.00 \\
\pm 7.74)\end{array}$ & & $\begin{array}{c}(10.0 \\
0+4.0 \\
8)\end{array}$ & $\begin{array}{c}(2.00 \pm 1 \\
.81)\end{array}$ & $\begin{array}{c}\left(12.00_{ \pm}\right. \\
5.89)\end{array}$ & $\stackrel{+}{\infty}$ & $\begin{array}{l}(8.00 \pm 2 \\
.85)\end{array}$ & $\begin{array}{c}(9.00 \pm 3 \\
.67)\end{array}$ & $\begin{array}{l}(17.00 \\
\pm 6.52)\end{array}$ & & \\
\hline \multirow{2}{*}{ Adress } & $\mathrm{U}$ & $(12.00 \pm 4.89)$ & $\begin{array}{c}(5.00 \pm \\
2.04)\end{array}$ & $\begin{array}{l}(17.00 \\
\pm 6.93)\end{array}$ & \multirow{2}{*}{ 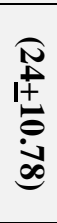 } & $\begin{array}{c}(9.00 \\
\pm 3.67 \\
)\end{array}$ & $\begin{array}{c}(3.00 \pm 1 \\
.22)\end{array}$ & $\begin{array}{c}(12.00 \pm \\
4.89)\end{array}$ & 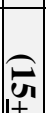 & $\begin{array}{l}(8.00 \pm 2 \\
.85)\end{array}$ & $\begin{array}{c}(8.00 \pm 2 \\
.85)\end{array}$ & $\begin{array}{c}(16.00 \\
\pm 5.7)\end{array}$ & \multirow{2}{*}{ 崩 } & \\
\hline & $\mathrm{R}$ & $(5.00 \pm 2.04)$ & $\begin{array}{c}(2.00 \pm \\
1.81)\end{array}$ & $\begin{array}{c}(7.00 \pm 3 \\
.85)\end{array}$ & & $\begin{array}{c}(3.00 \\
\pm 1.22 \\
)\end{array}$ & $\begin{array}{l}(0.00 \pm 0 \\
.00)\end{array}$ & $\begin{array}{c}(3.00 \pm 1 \\
22)\end{array}$ & 政 & $\begin{array}{l}(3.00 \pm 1 \\
.22)\end{array}$ & $\begin{array}{c}(2.00 \pm 1 \\
.81)\end{array}$ & $\begin{array}{c}(5.00 \pm 3 \\
.03)\end{array}$ & & \\
\hline \multirow{3}{*}{ Day /week } & 1 & $(0.00 \pm 0.00)$ & $\begin{array}{c}(0.00 \pm \\
0.00)\end{array}$ & $\begin{array}{c}(0.00 \pm 0 \\
.00)\end{array}$ & \multirow{3}{*}{ 芯 } & $\begin{array}{c}(0.00 \\
+0.00 \\
)\end{array}$ & $\begin{array}{l}(0.00 \pm 0 \\
.00)\end{array}$ & $\begin{array}{c}(0.00+0 \\
00)\end{array}$ & & $\begin{array}{c}(4.00 \pm 1 \\
.63)\end{array}$ & $\begin{array}{c}(1.00 \pm 0 \\
.40)\end{array}$ & $\begin{array}{c}(5.00 \pm 2 \\
.03)\end{array}$ & \multirow{3}{*}{ 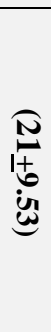 } & \\
\hline & 2 & $(2.00 \pm 1.81)$ & $\begin{array}{l}(2.00 \pm \\
1.81)\end{array}$ & $\begin{array}{c}(4.00 \pm 3 \\
.62)\end{array}$ & & $\begin{array}{c}(2.00 \\
\pm 1.81 \\
)\end{array}$ & $\begin{array}{l}(0.00 \pm 0 \\
.00)\end{array}$ & $\begin{array}{c}(2.00 \pm 1 \\
81)\end{array}$ & 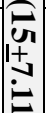 & $\begin{array}{c}(1.00 \pm 0 \\
.40)\end{array}$ & $\begin{array}{c}(2.00 \pm 1 \\
.81)\end{array}$ & $\begin{array}{c}(3.00 \pm 2 \\
.21)\end{array}$ & & \\
\hline & $>3$ & $(15.00 \pm 6.12)$ & $\begin{array}{l}(5.00 \pm \\
2.04)\end{array}$ & $\begin{array}{l}(20.00 \\
\pm 8.16)\end{array}$ & & $\begin{array}{c}(10.0 \\
0+4.0 \\
8)\end{array}$ & $\begin{array}{l}(3.00 \pm 1 \\
.22)\end{array}$ & $\begin{array}{c}(13.00 \pm \\
5.3)\end{array}$ & & $\begin{array}{l}(6.00 \pm 2 \\
.44)\end{array}$ & $\begin{array}{c}(7.00 \pm 2 \\
.85)\end{array}$ & $\begin{array}{l}(13.00 \\
\pm 5.29)\end{array}$ & & \\
\hline \multirow{2}{*}{ (hrs.)/ day } & $<12$ & $(15.00 \pm 6.12)$ & $\begin{array}{l}(5.00 \pm \\
2.04)\end{array}$ & $\begin{array}{l}(20.00 \\
\pm 8.16)\end{array}$ & \multirow{2}{*}{ 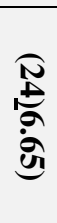 } & $\begin{array}{c}(10.0 \\
0 \pm 4.0 \\
8)\end{array}$ & $\begin{array}{l}(3.00 \pm 1 \\
.22)\end{array}$ & $\begin{array}{c}(13.00 \pm \\
5.3)\end{array}$ & 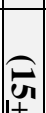 & $\begin{array}{l}(6.00 \pm 2 \\
.44)\end{array}$ & $\begin{array}{c}(8.00 \pm 2 \\
.85)\end{array}$ & $\begin{array}{c}(14.00 \\
\pm 5.29)\end{array}$ & \multirow{2}{*}{$\begin{array}{l}\underset{N}{+N} \\
\stackrel{+}{E}\end{array}$} & \\
\hline & $>13$ & $(2.00 \pm 1.81)$ & $\begin{array}{c}(2.00 \pm \\
1.81)\end{array}$ & $\begin{array}{c}(4.00 \pm 3 \\
.62)\end{array}$ & & $\begin{array}{c}(2.00 \\
\pm 1.81 \\
)\end{array}$ & $\begin{array}{l}(0.00 \pm 0 \\
.00)\end{array}$ & $\begin{array}{c}(2.00 \pm 1 \\
81)\end{array}$ & $\stackrel{-}{\Xi}$ & $\begin{array}{c}(5.00 \pm 2 \\
.04)\end{array}$ & $\begin{array}{c}(2.00 \pm 1 \\
.81)\end{array}$ & $\begin{array}{c}(7.00 \pm 3 \\
.85)\end{array}$ & & \\
\hline
\end{tabular}




\begin{tabular}{|c|c|c|c|c|c|c|c|c|c|c|c|c|c|}
\hline \multirow{3}{*}{ Purposes } & $\begin{array}{c}\text { Sci } \\
\text { enti } \\
\text { fic }\end{array}$ & $(3.00 \pm 1.22)$ & $\begin{array}{c}(2.00 \pm \\
1.81)\end{array}$ & $\begin{array}{c}(5.00 \pm 3 \\
.03)\end{array}$ & \multirow{3}{*}{ 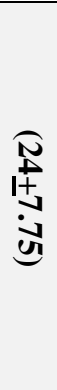 } & $\begin{array}{c}(4.00 \\
\pm 1.63 \\
)\end{array}$ & $\begin{array}{l}(0.00 \pm 0 \\
.00)\end{array}$ & $\begin{array}{c}(4.00 \pm 1 \\
63)\end{array}$ & \multirow{3}{*}{ 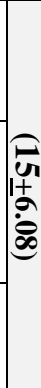 } & $\begin{array}{c}(6.00 \pm 2 \\
.44)\end{array}$ & $\begin{array}{c}(3.00 \pm 1 \\
.22)\end{array}$ & $\begin{array}{c}(9.00 \pm 3 \\
.65)\end{array}$ & \multirow{3}{*}{ 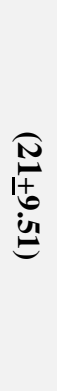 } \\
\hline & $\begin{array}{c}\text { Ent } \\
\text { erta } \\
\text { inin } \\
\mathrm{g}\end{array}$ & $(3.00 \pm 1.22)$ & $\begin{array}{c}(0.00 \pm \\
0.00)\end{array}$ & $\begin{array}{l}(3.00 \pm 1 \\
.22)\end{array}$ & & $\begin{array}{c}(1.00 \\
\pm 0.40 \\
)\end{array}$ & $\begin{array}{c}(1.00 \pm 0 \\
.40)\end{array}$ & $\begin{array}{c}(2.00 \pm 0 \\
.8)\end{array}$ & & $\begin{array}{c}(2.00 \pm 1 \\
.81)\end{array}$ & $\begin{array}{c}(1.00 \pm 0 \\
.40)\end{array}$ & $\begin{array}{c}(3.00 \pm 2 \\
.21)\end{array}$ & \\
\hline & $\begin{array}{c}\text { Bot } \\
\mathrm{h}\end{array}$ & $(11.00 \pm 4.49)$ & $\begin{array}{c}(5.00 \pm \\
2.04)\end{array}$ & $\begin{array}{l}(16.00 \\
\pm 6.53) \\
\end{array}$ & & $\begin{array}{c}(7.00 \\
\pm 2.85 \\
)\end{array}$ & $\begin{array}{c}(2.00 \pm 1 \\
.81)\end{array}$ & $\begin{array}{c}(9.00 \pm 3 \\
.65)\end{array}$ & & $\begin{array}{c}(3.00 \pm 1 \\
.22)\end{array}$ & $\begin{array}{c}(6.00 \pm 2 \\
.44)\end{array}$ & $\begin{array}{c}(9.00 \pm 3 \\
.65)\end{array}$ & \\
\hline \multirow{3}{*}{ Lighting level } & low & $(1.00 \pm 0.40)$ & $\begin{array}{l}(1.00 \pm \\
0.40)\end{array}$ & $\begin{array}{c}(2.00 \pm 0 \\
.8)\end{array}$ & \multirow{3}{*}{ 章 } & $\begin{array}{c}(1.00 \\
\pm 0.40 \\
)\end{array}$ & $\begin{array}{c}(0.00 \pm 0 \\
.00)\end{array}$ & $\begin{array}{c}(1.00 \pm 0 . \\
40)\end{array}$ & \multirow{3}{*}{ 光 } & $\begin{array}{c}(2.00 \pm 1 \\
.81)\end{array}$ & $\begin{array}{c}(1.00 \pm 0 \\
.40)\end{array}$ & $\begin{array}{c}(3.00 \pm 2 \\
.21)\end{array}$ & \multirow{3}{*}{ 疍 } \\
\hline & $\begin{array}{c}\mathrm{Me} \\
\mathrm{dia} \\
\mathrm{n}\end{array}$ & $(15.00 \pm 6.12)$ & $\begin{array}{c}(4.00 \pm \\
1.63)\end{array}$ & $\begin{array}{l}(19.00 \\
\pm 7.75)\end{array}$ & & $\begin{array}{c}(9.00 \\
\pm 3.67 \\
)\end{array}$ & $\begin{array}{c}(3.00 \pm 1 \\
.22)\end{array}$ & $\begin{array}{c}\left(12.00_{ \pm}\right. \\
4.89)\end{array}$ & & $\begin{array}{c}(7.00 \pm 2 \\
.85)\end{array}$ & $\begin{array}{c}(6.00 \pm 2 \\
.44)\end{array}$ & $\begin{array}{l}(13.00 \\
\pm 5.29)\end{array}$ & \\
\hline & $\begin{array}{c}\mathrm{Hig} \\
\mathrm{h}\end{array}$ & $(1.00 \pm 0.40)$ & $\begin{array}{c}(2.00 \pm \\
1.81)\end{array}$ & $\begin{array}{c}(3.00 \pm 2 \\
.21)\end{array}$ & & $\begin{array}{c}(2.00 \\
\pm 1.81 \\
)\end{array}$ & $\begin{array}{l}(0.00 \pm 0 \\
.00)\end{array}$ & $\begin{array}{c}(2.00 \pm 1 \\
81)\end{array}$ & & $\begin{array}{c}(2.00 \pm 1 \\
.81)\end{array}$ & $\begin{array}{l}(3.00 \pm 1 \\
.22)\end{array}$ & $\begin{array}{c}(5.00 \pm 3 \\
.03)\end{array}$ & \\
\hline \multirow{2}{*}{$\begin{array}{c}\text { Screen } \\
\text { protective }\end{array}$} & yes & $(2.00 \pm 1.81)$ & $\begin{array}{l}(2.00 \pm \\
1.81)\end{array}$ & $\begin{array}{c}(4.00 \pm 3 \\
.62)\end{array}$ & \multirow{2}{*}{ 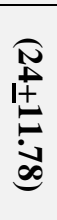 } & $\begin{array}{c}(3.00 \\
\pm 1.22 \\
)\end{array}$ & $\begin{array}{l}(0.00 \pm 0 \\
.00)\end{array}$ & $\begin{array}{c}(3.00 \pm 1 \\
22)\end{array}$ & \multirow{2}{*}{ 它 } & $\begin{array}{c}(3.00 \pm 1 \\
.22)\end{array}$ & $\begin{array}{l}(3.00 \pm 1 \\
.22)\end{array}$ & $\begin{array}{c}(6.00 \pm 2 \\
.44)\end{array}$ & \multirow{2}{*}{ 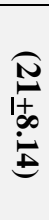 } \\
\hline & No & $(15.00 \pm 6.12)$ & $\begin{array}{c}(5.00 \pm \\
2.04)\end{array}$ & $\left(20 \pm \frac{ \pm}{6} 8.1\right.$ & & $\begin{array}{c}(9.00 \\
+3.67 \\
)\end{array}$ & $\begin{array}{c}(3.00 \pm 1 \\
.22)\end{array}$ & $\begin{array}{c}\left(12.00_{ \pm}\right. \\
4.89)\end{array}$ & & $\begin{array}{c}(8.00 \pm 2 \\
.85)\end{array}$ & $\begin{array}{c}(7.00 \pm 2 \\
.85)\end{array}$ & $\begin{array}{l}(15.00 \\
\pm 5.7)\end{array}$ & \\
\hline \multirow{2}{*}{$\begin{array}{l}\text { Sreen Distance } \\
\text { from eye }(\mathrm{cm})\end{array}$} & $<10$ & $(3.00 \pm 1.22)$ & $\begin{array}{c}(2.00 \pm \\
1.81)\end{array}$ & $\begin{array}{c}(5.00 \pm 3 \\
.03)\end{array}$ & \multirow{2}{*}{ 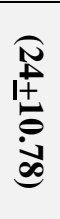 } & $\begin{array}{c}(2.00 \\
+1.81 \\
)\end{array}$ & $\begin{array}{c}(0.00 \pm 0 \\
.00)\end{array}$ & $\begin{array}{c}(2.00 \pm 1 \\
81)\end{array}$ & \multirow{2}{*}{ 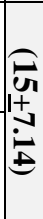 } & $\begin{array}{c}(4.00 \pm 1 \\
.63)\end{array}$ & $\begin{array}{l}(3.00 \pm 1 \\
.22)\end{array}$ & $\begin{array}{c}(7.00 \pm 2 \\
.85)\end{array}$ & \multirow{2}{*}{ 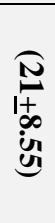 } \\
\hline & $>10$ & $(14.00 \pm 5.71)$ & $\begin{array}{c}(5.00 \pm \\
2.04)\end{array}$ & $\begin{array}{l}(19.00 \\
\pm 7.75)\end{array}$ & & $\begin{array}{c}(10.0 \\
0 \pm 4.0 \\
8)\end{array}$ & $\begin{array}{l}(3.00 \pm 1 \\
.22)\end{array}$ & $\begin{array}{c}\left(13.00_{ \pm}\right. \\
5.33)\end{array}$ & & $\begin{array}{l}(7.00 \pm 2 \\
.85)\end{array}$ & $\begin{array}{l}(7.00 \pm 2 \\
.85)\end{array}$ & $\begin{array}{c}(14.00 \\
\pm 5.7)\end{array}$ & \\
\hline \multirow{3}{*}{$\begin{array}{c}\text { Place lighting } \\
\text { level }\end{array}$} & low & $(3.00 \pm 1.22)$ & $\begin{array}{c}(1.00 \pm \\
0.40)\end{array}$ & $\begin{array}{c}(4.00 \pm 1 \\
.62)\end{array}$ & \multirow{3}{*}{ 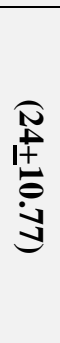 } & $\begin{array}{l}(0.00 \\
\pm 0.00 \\
\end{array}$ & $\begin{array}{l}(0.00 \pm 0 \\
.00)\end{array}$ & $\begin{array}{c}(0.00 \pm 0 . \\
00)\end{array}$ & \multirow{3}{*}{ 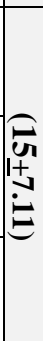 } & $\begin{array}{c}(2.00 \pm 1 \\
.81)\end{array}$ & $\begin{array}{c}(0.00 \pm 0 \\
.00)\end{array}$ & $\begin{array}{c}(2.00 \pm 1 \\
.81)\end{array}$ & \multirow{3}{*}{ 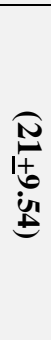 } \\
\hline & $\begin{array}{c}\mathrm{Me} \\
\mathrm{dia} \\
\mathrm{n}\end{array}$ & $(14.00 \pm 5.71)$ & $\begin{array}{c}(4.00 \pm \\
1.63)\end{array}$ & $\begin{array}{l}(18.00 \\
\pm 7.34)\end{array}$ & & $\begin{array}{c}(10.0 \\
0+4.0 \\
8)\end{array}$ & $\begin{array}{l}(3.00 \pm 1 \\
.22)\end{array}$ & $\begin{array}{c}\left(13.00_{ \pm}\right. \\
5.3)\end{array}$ & & $\begin{array}{l}(6.00 \pm 2 \\
.44)\end{array}$ & $\begin{array}{l}(6.00 \pm 2 \\
.44)\end{array}$ & $\begin{array}{l}(12.00 \\
+4.88)\end{array}$ & \\
\hline & $\underset{\mathrm{h}}{\mathrm{Hig}}$ & $(0.00 \pm 0.00)$ & $\begin{array}{c}(2.00 \pm \\
1.81)\end{array}$ & $\begin{array}{l}(2.00 \pm 1 \\
.81)\end{array}$ & & $\begin{array}{c}(2.00 \\
\pm 1.81 \\
)\end{array}$ & $\begin{array}{c}(0.00 \pm 0 \\
.00)\end{array}$ & $\begin{array}{c}(2.00 \pm 1 \\
81)\end{array}$ & & $\begin{array}{l}(3.00 \pm 1 \\
.22)\end{array}$ & $\begin{array}{l}(4.00 \pm 1 \\
.63)\end{array}$ & $\begin{array}{c}(7.00 \pm 2 \\
.85)\end{array}$ & \\
\hline \multirow[b]{2}{*}{ Lighting type } & $\begin{array}{c}\text { Nor } \\
\text { mal } \\
\text { ligh } \\
\mathrm{t}\end{array}$ & $(6.00 \pm 2.44)$ & $\begin{array}{c}(3.00 \pm \\
1.22)\end{array}$ & $\begin{array}{c}(9.00 \pm \\
3.66)\end{array}$ & \multirow{2}{*}{ 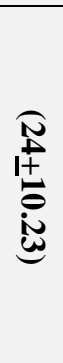 } & $\begin{array}{c}(4.00 \\
+1.63 \\
)\end{array}$ & $\begin{array}{c}(1.00 \pm 0 \\
.40)\end{array}$ & $\begin{array}{c}(5.00 \pm 2 \\
.03)\end{array}$ & \multirow{2}{*}{ 光 } & $\begin{array}{l}(7.00 \pm 2 \\
.85)\end{array}$ & $\begin{array}{l}(4.00 \pm 1 \\
.63)\end{array}$ & $\begin{array}{l}(11.00 \\
\pm 4.48)\end{array}$ & \multirow{2}{*}{ 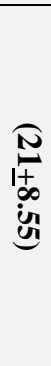 } \\
\hline & $\begin{array}{c}\text { Eco } \\
\text { no } \\
\text { mic } \\
\text { ligh } \\
\text { t }\end{array}$ & $(11.00 \pm 4.49)$ & $\begin{array}{c}(4.00 \pm \\
1.63)\end{array}$ & $\begin{array}{l}(15.00 \\
\pm 6.57)\end{array}$ & & $\begin{array}{c}(8.00 \\
\pm 2.85 \\
)\end{array}$ & $\begin{array}{l}(2.00 \pm 1 \\
.81)\end{array}$ & $\begin{array}{c}\left(10.00_{ \pm}\right. \\
4.66)\end{array}$ & & $\begin{array}{l}(4.00 \pm 1 \\
.63)\end{array}$ & $\begin{array}{c}(6.00 \pm 2 \\
.44)\end{array}$ & $\begin{array}{c}(10.00 \\
\pm 4.07)\end{array}$ & \\
\hline \multirow{3}{*}{ Lights no. } & 1 & $(5.00 \pm 2.04)$ & $\begin{array}{c}(1.00 \pm \\
0.40)\end{array}$ & $\begin{array}{c}(6.00 \pm 2 \\
.44)\end{array}$ & \multirow{3}{*}{ 莽 } & $\begin{array}{c}(2.00 \\
\pm 1.81 \\
)\end{array}$ & $\begin{array}{l}(2.00 \pm 1 \\
.81)\end{array}$ & $\begin{array}{c}(4.00 \pm 3 \\
.62)\end{array}$ & \multirow{3}{*}{ 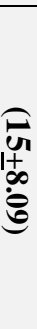 } & $\begin{array}{l}(4.00 \pm 1 \\
.63)\end{array}$ & $\begin{array}{c}(3.00 \pm 1 \\
.22)\end{array}$ & $\begin{array}{c}(7.00 \pm 2 \\
.85)\end{array}$ & \multirow{3}{*}{ 莫 } \\
\hline & 2 & $(8.00 \pm 2.85)$ & $\begin{array}{c}(2.00 \pm \\
1.81)\end{array}$ & $\begin{array}{l}(10.00 \\
\pm 4.66)\end{array}$ & & $\begin{array}{c}(6.00 \\
\pm 2.44 \\
)\end{array}$ & $\begin{array}{c}(1.00 \pm 0 \\
.40)\end{array}$ & $\begin{array}{c}(7.00 \pm 2 \\
.84)\end{array}$ & & $\begin{array}{c}(5.00 \pm 2 \\
.04)\end{array}$ & $\begin{array}{c}(2.00 \pm 1 \\
.81)\end{array}$ & $\begin{array}{c}(7.00 \pm 3 \\
.85)\end{array}$ & \\
\hline & $>3$ & $(4.00 \pm 1.63)$ & $\begin{array}{l}(4.00 \pm \\
1.63)\end{array}$ & $\begin{array}{c}(8.00 \pm 3 \\
.26)\end{array}$ & & $\begin{array}{c}(4.00 \\
+1.63 \\
)\end{array}$ & $\begin{array}{c}(0.00 \pm \\
0.00)\end{array}$ & $\begin{array}{c}(4.00 \pm 1 \\
63)\end{array}$ & & $\begin{array}{c}(2.00 \pm 1 \\
.81)\end{array}$ & $\begin{array}{c}(5.00 \pm 2 \\
.04)\end{array}$ & $\begin{array}{c}(7.00 \pm 3 \\
.85)\end{array}$ & \\
\hline
\end{tabular}




\begin{tabular}{|c|c|c|c|c|c|c|c|c|c|c|c|c|c|c|}
\hline \multirow{3}{*}{ Using place } & $\begin{array}{l}\text { ho } \\
\text { me }\end{array}$ & \multicolumn{2}{|c|}{$(13.00 \pm 5.30)$} & $\begin{array}{l}(5.00 \pm \\
2.04)\end{array}$ & $\begin{array}{l}(18.00 \\
\pm 7.34) \\
\end{array}$ & \multirow{3}{*}{ 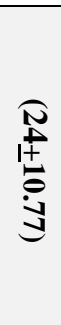 } & $\begin{array}{c}(8.00 \\
\pm 2.85 \\
\quad) \\
\end{array}$ & $\begin{array}{l}(2.00 \pm 1 \\
.81)\end{array}$ & $\begin{array}{c}(10.00 \pm \\
4.66)\end{array}$ & & $\begin{array}{l}(7.00 \pm 2 \\
.85)\end{array}$ & $\begin{array}{l}(7.00 \pm 2 \\
.85)\end{array}$ & $\begin{array}{c}(14.00 \\
\pm 5.7)\end{array}$ & \multirow{3}{*}{ 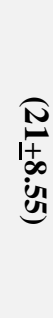 } \\
\hline & $\begin{array}{l}\text { Off } \\
\text { ice }\end{array}$ & \multicolumn{2}{|c|}{$(3.00 \pm 1.22)$} & $\begin{array}{l}(2.00 \pm \\
1.81)\end{array}$ & $\begin{array}{c}5.00 \pm 3 . \\
03)\end{array}$ & & $\begin{array}{l}(1.00 \\
\pm 0.70\end{array}$ & $\begin{array}{l}(1.00 \pm 0 \\
.40)\end{array}$ & $\begin{array}{l}(2.00 \pm 1 \\
.1)\end{array}$ & \multirow[t]{2}{*}{ 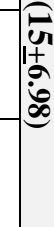 } & $\begin{array}{l}(4.00 \pm 1 \\
.63)\end{array}$ & $\begin{array}{l}(3.00 \pm 1 \\
.22)\end{array}$ & $\begin{array}{c}(7.00 \pm 2 \\
.85)\end{array}$ & \\
\hline & out & \multicolumn{2}{|c|}{$(1.00 \pm 0.40)$} & $\begin{array}{c}(0.00 \pm \\
0.00)\end{array}$ & $\begin{array}{l}(1.00 \pm 0 \\
.40)\end{array}$ & & $\begin{array}{c}(3.00 \\
\pm 1.22 \\
)\end{array}$ & $\begin{array}{l}(0.00 \pm 0 \\
.00)\end{array}$ & $\begin{array}{c}(3.00 \pm 1 . \\
22)\end{array}$ & & $\begin{array}{l}(0.00 \pm 0 \\
.00)\end{array}$ & $\begin{array}{l}(0.00 \pm 0 \\
.00)\end{array}$ & $\begin{array}{l}(0.00 \pm 0 \\
.00)\end{array}$ & \\
\hline \multirow{2}{*}{$\begin{array}{l}\text { Lighting } \\
\text { direction }\end{array}$} & $\begin{array}{c}\text { Fro } \\
\text { nt }\end{array}$ & \multicolumn{2}{|c|}{$(10.00 \pm 4.08)$} & $\begin{array}{c}(4.00 \pm \\
1.63)\end{array}$ & $\begin{array}{l}(14.00 \\
\pm 5.71) \\
\end{array}$ & \multirow{2}{*}{ 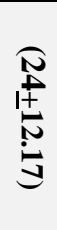 } & $\begin{array}{c}(7.00 \\
\pm 2.85 \\
\quad)\end{array}$ & $\begin{array}{l}(1.00 \pm 0 \\
.40)\end{array}$ & $\begin{array}{c}(8.00 \pm 3 \\
.25)\end{array}$ & \multirow{2}{*}{ 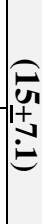 } & $\begin{array}{l}(6.00 \pm 2 \\
.44)\end{array}$ & $\begin{array}{l}(6.00 \pm 2 \\
.44)\end{array}$ & $\begin{array}{l}(12.00 \\
\pm 4.88)\end{array}$ & \multirow{2}{*}{ 孞 } \\
\hline & $\begin{array}{l}\text { beh } \\
\text { ind }\end{array}$ & \multicolumn{2}{|c|}{$(7.00 \pm 2.84)$} & $\begin{array}{l}(3.00 \pm \\
2.21)\end{array}$ & $\begin{array}{l}(10.00 \\
\pm 6.46)\end{array}$ & & $\begin{array}{c}(5.00 \\
\pm 2.04 \\
)\end{array}$ & $\begin{array}{c}(2.00 \pm 1 \\
.81)\end{array}$ & $\begin{array}{c}(7.00 \pm 3 \\
.85)\end{array}$ & & $\begin{array}{l}(5.00 \pm 3 \\
.03)\end{array}$ & $\begin{array}{l}(4.00 \pm 1 \\
.62)\end{array}$ & $\begin{array}{c}(9.00 \pm 4 \\
.65)\end{array}$ & \\
\hline \multirow{2}{*}{ Eye allergy } & yes & \multicolumn{2}{|c|}{$(4.00 \pm 1.63)$} & $\begin{array}{l}(2.00 \pm \\
1.81)\end{array}$ & $\begin{array}{c}(6.00 \pm 3 \\
.44)\end{array}$ & \multirow{2}{*}{ 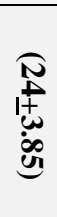 } & $\begin{array}{l}(4.00 \\
\pm 1.63 \\
\end{array}$ & $\begin{array}{l}(1.00 \pm 0 \\
.40)\end{array}$ & $\begin{array}{l}(5.00 \pm 2 \\
.03)\end{array}$ & in & $\begin{array}{l}(3.00 \pm 1 \\
.22)\end{array}$ & $\begin{array}{l}(5.00 \pm 2 \\
.04)\end{array}$ & $\begin{array}{l}(8.00 \pm 3 \\
.26)\end{array}$ & \multirow{2}{*}{ 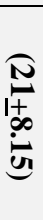 } \\
\hline & No & \multicolumn{2}{|c|}{$(13.00 \pm 5.30)$} & $\begin{array}{l}(5.00 \pm \\
2.04)\end{array}$ & $\begin{array}{l}(18.00 \\
\pm 7.34)\end{array}$ & & $\begin{array}{c}(8.00 \\
\pm 2.85 \\
)\end{array}$ & $\begin{array}{l}(2.00 \pm 1 \\
.81)\end{array}$ & $\begin{array}{c}(10.00 \pm \\
4.66)\end{array}$ & क्ष & $\begin{array}{l}(8.00 \pm 2 \\
.85)\end{array}$ & $\begin{array}{l}(5.00 \pm 2 \\
.04)\end{array}$ & $\begin{array}{l}(13.00 \\
\pm 4.89)\end{array}$ & \\
\hline \multirow{2}{*}{$\begin{array}{l}\text { Week sighting } \\
\text { familian } \\
\text { history }\end{array}$} & yes & \multicolumn{2}{|c|}{$(3.00 \pm 1.22)$} & $\begin{array}{l}(5.00 \pm \\
2.04)\end{array}$ & $\begin{array}{l}(8.00 \pm \\
3.26)\end{array}$ & \multirow{2}{*}{ 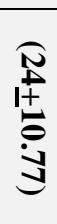 } & $\begin{array}{l}(3.00 \\
\pm 1.22 \\
)\end{array}$ & $\begin{array}{l}(0.00 \pm 0 \\
.00)\end{array}$ & $\begin{array}{c}(3.00 \pm 1 . \\
22)\end{array}$ & & $\begin{array}{l}(5.00 \pm 2 \\
.04)\end{array}$ & $\begin{array}{l}(6.00 \pm 2 \\
.44)\end{array}$ & $\begin{array}{l}(11.00 \\
\pm 4.48)\end{array}$ & \multirow{2}{*}{ 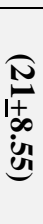 } \\
\hline & No & \multicolumn{2}{|c|}{$(14.00 \pm 5.7)$} & $\begin{array}{c}(2.00 \pm \\
1.81)\end{array}$ & $\begin{array}{l}(16.00 \\
\pm 7.51)\end{array}$ & & $\begin{array}{c}(9.00 \\
\pm 3.67 \\
\quad)\end{array}$ & $\begin{array}{l}(3.00 \pm 1 \\
.22)\end{array}$ & $\begin{array}{c}(12.00 \pm \\
4.89)\end{array}$ & & $\begin{array}{l}(6.00 \pm 2 \\
.44)\end{array}$ & $\begin{array}{l}(4.00 \pm 1 \\
.63)\end{array}$ & $\begin{array}{l}(10.00 \\
\pm 4.07)\end{array}$ & \\
\hline \multirow{2}{*}{$\begin{array}{l}\text { Glass wearing } \\
\text { putting on }\end{array}$} & yes & \multicolumn{2}{|c|}{$(7.00 \pm 2.85)$} & $\begin{array}{c}\left(4.00_{ \pm}\right. \\
1.63)\end{array}$ & $\begin{array}{l}(11.00 \\
\pm 4.48) \\
\end{array}$ & \multirow{2}{*}{ 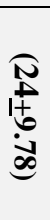 } & $\begin{array}{c}(4.00 \\
\pm 1.63 \\
\quad)\end{array}$ & $\begin{array}{l}(1.00 \pm 0 \\
.40)\end{array}$ & $\begin{array}{l}(5.00 \pm 2 \\
.03)\end{array}$ & $\vec{\theta}_{1+}$ & $\begin{array}{l}(7.00 \pm 2 \\
.85)\end{array}$ & $\begin{array}{l}(7.00 \pm 2 \\
.85)\end{array}$ & $\begin{array}{c}(14.00 \\
\pm 5.7)\end{array}$ & \multirow{2}{*}{ 蛋 } \\
\hline & No & \multicolumn{2}{|c|}{$(10.00 \pm 4.08)$} & $\begin{array}{l}(3.00 \pm \\
1.22)\end{array}$ & $\stackrel{(13+5.3}{)}$ & & $\begin{array}{c}(8.00 \\
\pm 2.85 \\
\quad)\end{array}$ & $\begin{array}{c}(2.00 \pm 1 \\
.81)\end{array}$ & $\begin{array}{c}(10.00 \pm \\
4.66)\end{array}$ & 尚 & $\begin{array}{c}(4.00 \pm 1 \\
.63)\end{array}$ & $\begin{array}{l}(3.00 \pm 1 \\
.22)\end{array}$ & $\begin{array}{l}(7.00 \pm 2 \\
.85)\end{array}$ & \\
\hline \multirow{4}{*}{$\begin{array}{c}\text { Opetical } \\
\text { putting time }\end{array}$} & $\begin{array}{l}\text { Bef } \\
\text { ore }\end{array}$ & 1 & $\begin{array}{l}(2.00 \pm \\
1.81)\end{array}$ & $\begin{array}{c}(0.00 \pm \\
0.00)\end{array}$ & $\begin{array}{c}(2.00 \pm 1 \\
.81)\end{array}$ & \multirow{2}{*}{ 垈 } & $\begin{array}{l}(3.00 \\
\pm 1.22 \\
)\end{array}$ & $\begin{array}{c}(0.00 \pm 0 \\
.00)\end{array}$ & $\begin{array}{c}(3.00 \pm 1 . \\
22)\end{array}$ & $f$ & $\begin{array}{l}(2.00 \pm 1 \\
.81)\end{array}$ & $\begin{array}{l}(2.00 \pm 1 \\
.81)\end{array}$ & $\begin{array}{c}(4.00 \pm 3 \\
.62)\end{array}$ & \multirow{2}{*}{ 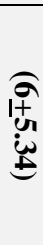 } \\
\hline & $\begin{array}{c}\text { ng( } \\
\text { yrs. } \\
\text { ) }\end{array}$ & $>1$ & $\begin{array}{c}(0.00 \pm \\
0.00)\end{array}$ & $\begin{array}{c}\left(3.00_{ \pm}\right. \\
1.22)\end{array}$ & $\begin{array}{c}(3.00 \pm 1 \\
.22)\end{array}$ & & $\begin{array}{c}(1.00 \\
\pm 0.40 \\
\quad)\end{array}$ & $\begin{array}{c}(0.00 \pm 0 \\
.00)\end{array}$ & $\begin{array}{c}(1.00 \pm 0 . \\
40)\end{array}$ & స్తి & $\begin{array}{l}(2.00 \pm 1 \\
.81)\end{array}$ & $\begin{array}{l}(0.00 \pm 0 \\
.00)\end{array}$ & $\begin{array}{c}(2.00 \pm 1 \\
.81)\end{array}$ & \\
\hline & \multirow{2}{*}{$\begin{array}{l}\text { Aft } \\
\text { er } \\
\text { usi } \\
\text { ng( } \\
\text { yrs. }\end{array}$} & 1 & $\begin{array}{c}(2.00 \pm \\
1.81)\end{array}$ & $\begin{array}{c}(1.00 \pm \\
0.40)\end{array}$ & $\begin{array}{c}(3.00 \pm 2 \\
.21)\end{array}$ & \multirow{2}{*}{ 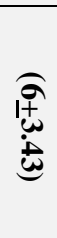 } & $\begin{array}{c}(0.00 \\
\pm 0.00 \\
\quad) \\
\end{array}$ & $\begin{array}{c}(1.00 \pm 0 \\
.40)\end{array}$ & $\begin{array}{c}(1.00 \pm 0 . \\
40)\end{array}$ & & $\begin{array}{l}(1.00 \pm 0 \\
.40)\end{array}$ & $\begin{array}{l}(2.00 \pm 1 \\
.81)\end{array}$ & $\begin{array}{c}(3.00 \pm 2 \\
.21)\end{array}$ & \multirow{2}{*}{ 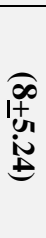 } \\
\hline & & $>1$ & $\begin{array}{c}(3.00 \pm \\
1.22)\end{array}$ & $\begin{array}{c}(0.00 \pm \\
0.00)\end{array}$ & $\begin{array}{c}(3.00 \pm 1 \\
.22)\end{array}$ & & $\begin{array}{l}(0.00 \\
\pm 0.00\end{array}$ & $\begin{array}{l}(0.00 \pm 0 \\
.00)\end{array}$ & $\begin{array}{c}(0.00 \pm 0 . \\
00)\end{array}$ & 草 & $\begin{array}{l}(2.00 \pm 1 \\
.81)\end{array}$ & $\begin{array}{l}(3.00 \pm 1 \\
.22)\end{array}$ & $\begin{array}{c}(5.00 \pm 3 \\
.03)\end{array}$ & \\
\hline
\end{tabular}

- m:male;f:female;u:urban;r:ruler;s:single;ma:marriage

- Different capital refer to significant differences between groups horizontally $(\mathrm{P} \leq 0.05)$.

-The values represent Mean \pm SE 


\section{5- Conclusions}

1. Using computers and smart devices of students more than employers.

2. There are eye sensitive problems shown in such of employers and students.

3. Appearing many status of computer vision syndrome in employers more than students.

4. Appearing different effecting of eye glass wearing of students and employers that they were utilizing different computers and smart devices with some risk factors according to others.

5. It is found assuring correlations among different risk factors, but shown to employers greater than students.

\section{CONFLICT OF INTERESTS:}

\section{There are no conflicts of interest.}

\section{6- References}

[1] A. Barar, ID. Apatachioaie, C. Apatachioaie, L. Marceanu-Brasov and Oftalmologia."Ophthalmologist and"computer vision syndrome". Article in Romanian. 51(3):104-9. PMID:18064965.2007.

[2] S .IJmker, M.A. Huysmans, B.M. Blatter, A.J. Van Der Beek, M.W. Van and P.M. Bongers. "Should office workers spend fewer hours at their computer? A systematic review of the literature". Occup Environ Med.;64(4):211-22. http://dx.doi.org/10.1136/ oem.2006.026468. 2007.

[3] F. Gerr , C.P. Monteilh and M. Marcus. "Keyboard use and musculoskeletal outcomes among computer users". J Occup Rehabil. 16(3):265-77.http://dx.doi.org/10.1007/ s10926-006-9037-0. 2006.

[4] V.Woods, S. Hastings and P. Buckle, et al."Development of nonkeyboard input device checklists through assessments”. Appl Ergon. 34:511 - 519. 2003

[5] J. Bali, N. Navin and B.R. Thakur. "Computer vision syndrome: A study of the knowledge, attitudes and practices in Indian ophthalmologists". Indian J Ophthalmol. 55:289-94. 2007.

[6] T.Schlote, G. Kadner and N. Freudenthaler. "Marked reduction and distinct patterns of eye blinking in patients with moderately dry eyes during video display terminal use". Graefes Arch Clin Exp Ophthalmol. DOI:10107/ s00417 10003-0845-z. 2004.

[7] J. Wahlström.’Ergonomics, musculoskeletal disorders and computer work”. Occup Med. 55:168-176. 2005.

[8] W.A. Latko, T.J. Armstrong, A. Franzblau and et al. "Crosssectional study of the relationship between repetitive work and the prevalence of upper limb musculoskeletal disorders".Am J Ind Med.;36:248- 259. 1999.

[9] B.A. Silverstein, L.J. Fine and T.J. Armstrong. "Occupational factors and carpal tunnel syndrome".Am J Ind Med. 11:343-358. 1987.

[10] M. Feuerstein, T. Armstrong, P Hickey and et al." Computer keyboard force and upper extremity symptoms". $J$ Occup Environ Med;39:1144- 1153. 1997.

[11] S. Statistics."The working environment: Statistics Sweden". Report No. AM68SM0201. 2002.

[12] M. Waersted, T.N. Hanvold and K.B. Veiersted. "Computer work and musculoskeletal disorders of the neck and upper extremity: a systematic review”. BMC Musculoskelet Disord. 11:79. http://dx.doi.org/10.1186/1471-2474-1179. 2010.

[13] S. Tamez Gonzalez, L. Ortiz-Hernandez, S. Martinez-Alcantara, I. Mendez-Ramirez. "Risks and health problems caused by the use of video terminals". Salud Publica Mex. 45:171-80. 2003.

[14] G.R. MacKinnon. "A decade of laptop computers: The impact on the pedagogy of university faculty". Journal of Instruction Delivery Systems.;21:7-20. 2007.

[15] H. Johansson and P. Sojka. "Pathophysiological mechanisms involved in genesis and spread of muscular tension in occupational muscle pain and in chronic musculoskeletal pain syndromes: a hypothesis. Ha"gg GM. Static work load and occupational myalgia: a new explanation model. In: Andersen PA, Hobart DJ". Med Hypotheses. 35 : 28.196 203. 1991.

[16] P.W. Johnson."The Development, Characterization and Implementation of a Technique to Measure Muscle Fatigue During Computer Use". Berkeley: University of California, 1998.

[17] G. Sjøgaard, U. Lundberg and R. Kadefors. "The role of muscle activity and mental load in the development of pain and degenerative processes at the muscle cell level during computer work".Eur J Appl Physiol. 83:99 - 105. 2000.

[18] M. Hagberg. "Occupational musculoskeletal stress and disorders of the neck and shoulder: a review of possible pathophysiology”.Int Arch Occup Environ Health. 53: 269- 278. 1984.

[19]B.P. Bernard. "Ed. Musculoskeletal Disorders and Workplace Factors: A Critical Review of Epidemiological Evidence for Work-Related Musculoskeletal Disorders of the Neck, Upper Extremity, and Low Back". 2nd edn. Cincinnati: National Institute for Occupational Safety and Health (NIOSH), 1997. 
[20] L. Punnett and U. Bergqvist. "Visual display unit work and upper extremity musculoskeletal disorders". Stockholm: National Institute for Working Life, 1997.

[21] V.J. Rideout, U.G. Foehr and D.F. Roberts. "Generation $\mathrm{M}^{2}$. Media in the Lives of 8- to 18-Year Olds". A Kaiser Family Foundation Study. The Henry J. Kaiser Family Foundation: Menlo Park, CA, 2010.

[22] A. Aara ${ }^{\circ}$ s, G. Horgen, H.H. Bjørset and et al. "Musculoskeletal, visual and psychosocial stress in VDU operators before and after multidisciplinary ergonomic interventions. A 6 years prospective study- part II". Appl Ergon. 32: 559571. 2001.

[23] K.I. Fostervold. "VDU work with downward gaze: the emperor's new clothes or scientifically sound?". Int J Ind Ergonomics. 31:161- 167. 2003.

[24] J. Faucett, D. Rempel. "VDT-related musculoskeletal symptoms: Interactions between work posture and psychosocial work factors". AmJ Ind Med. 26:597-612. 1994.

[25] P.M. Bongers, C.R. De Winter, M.A.J Kompier and V.H. Hildebrandt. "Psychosocial factors at work and musculoskeletal disease". Scand J Work Environ Health. 19:297-312. 1993.

[26] W. Wayne Daniel. "Biostatic Basic Concepts and Methdology for the Health Sciences, "John Wiley \& Sons (Asia) Pte Ltd. 2010.

[27] G. Betram, B. Susan and J.Anthony. University of California, San Francisco. ppt. 113, 661t. 2009.

[28] J.V. Danoff, eds."Electromyographical Kinesiology”. Amsterdam: Elsevier. 141- 144. 1991.

[29] Computer vision syndrome (CVS). "American Optometric Association". Available from: http://www.aoa.org/x5374. xml. [Last cited on 2012 Apr 14].

ألاهداف: الاستقاء حول اعداد المستخدمين من الطلبة والموظفين المتأثزين بالاستخدام المتكرر للحاسبات والاجهزة الذكية, بالاضافة الى ذلك اجر اء دراسة مقارنة بينهم من خلال معرفة العلاقة مع مختلف العو امل الخطرة وتأثيرر اتها عليه. المواد وطرائق العدل: نفذت هذة الدر اسة على أفر اد قد تم تقسيمهم الى مجموعتين, المجموعة الاولى تضمنت [) مر احل من الكلية, اما المجموعة الثانية كانت [(60 individuals; 40 male, 20 female)], وجميعهم استخدموا الحاسبات والاجهزة الذكية خلال سنة در اسية واحدة (from January $1^{\text {st }} 2016$ to August 15 2017). هذة الدراسة تضمنت بحث العلاقة الاستعمال

المتعدد و المتكرر للحاسبات والاجهزة الذكية مع مختلف العوامل الخطرة. تلك البيانات تم تحليلها احصائياً بواسطة -ANOVA ) : اقل أو تساوي analysis of variance - two ways analyze; Mean \pm (pE) اظهرت النتائج فروقات معنوية في الطلبة المستخدمين للحاسوب والاجهزة الذكية حيث كانت هنالك اعداد قليلة من المستخدمين قد عانوا من التأثز بالرؤية وبمختلف الاعمار والاجناس. ان الاستخدام المطول للاجهزة ولمختلف الاغر اض لم يكن له ابي تأثثر فيما يخص تأثر العين للمستخمين من الطلاب. لوحظ بان ارتداء النظارات الطبية في الذكور كان اكثر من الاناث. من جانب اخر ان الاعمار التي تتر اوح مابين (ع ب-rrr) حصلت فيها اعلى معدلات في استخدام الحاسوب والاجهزة الذكية, وكذلك فان لحساسية العيون تأثثر اكبر من بين العوامل الخطرة مقارنة بعدد اوقات الاستخدام ذات المعدلات القليلة ـ بينت هذة النتاىج تفوق الذكور بالمفارنة مع الاناث في مجال استخدام الاجهزة المتتوزعة, لذا ومن خلال التتوع في اعداد الطلبة التي تمت ملاحظتها وخصوصا التاثير في رؤية العين والك عند

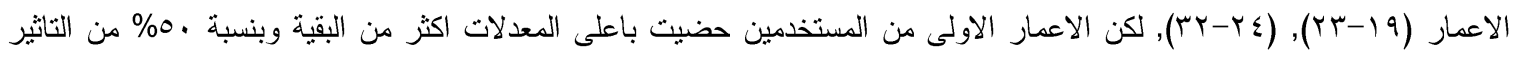
في مجال الرؤية للمستخدمين الطلبة, في حين المجموعة الثانية من الاعمار بلغت ثقريبا ( بس.بس \% ). ان النتائج التي وجدت للموظفين الذين كانوا قد استخدموالحاسوب والاجهزة الذكية اظهرت فيها فروقات معنوية (0.05 \ي) بالمقارنة مع النتائج السابقة للطلبة, حيث لوحظ اعداع كبيرة منهم كانوا يعانون من التأثر الرؤية لديهم نتيجة الاستخدام المفرط للاجهة المختلفة. ان المستخدمين من الموظفين الذين لديهم اعمار تثراوح (اب-.ـ) و لاغر اض مختلفة (علمية, ترفيهية او كلاهما) كانت لهم علاقة ذات تأثنر معنوي مع احداث الضرر فيما يخص الرؤية لاى المستخدمين بالمقارنة مع العوامل الخطرة الاخرى, بينما كان الاستخدام من قبل الموظفين تحت اعداد مختلفة من مصادر الاضوية اظهرت تأثير اقل, وكذلك لم تكن هنالك اي تاثير معنوي لارتداء النظارات الطبية من قبل المستخدمين للاجزةة, وان تأثير هذا العامل الاخير على مجمل اعداد الموضفين الذين يرتدون النظارات الطبية كان لها تاثير معنوي 
(p ب.05) المرتدين للنظارات الطبية من الموظفين المستخدمين للحاسبات والاجهزة الذكية على حد سواء وهذة تدل عل مدى استخدام الجهزة

$$
\text { وضر اوتها عليهم و التي كانت بنسبة (ع هـ هـ). }
$$

الاستنتاجات: ان استخدام الحاسبات والاججزة الذكية من قبل الطلاب كان أكثر مقارنة بُّلموظفين. هنالك علاقة وثيقة ارتداء النظارات الطيية مع مختلف العوامل الخطرة التي يتعرض لها المستخدمون من الطلاب والموظفين. ان العلاقة الوثيقة للتأثيرات التي وجدت بين مختلف العوامل الخطرة كانت أكثر في المستخدمين الموظفين بالمقارنة مع الطلاب. الكلمات الدالة: حاسبات, اجزة ذكية, موظفون, طلاب, عوامل خطرة, متلازمة رؤية الحاسبات. 